\title{
Drivers for construction stakeholders to adopt smart contracts
}

\author{
Kerim Koc* iD, Asli Pelin Gurgun iD \\ Yildiz Technical University, Civil Engineering Department, Istanbul, Turkey
}

\begin{abstract}
Construction contracts are always regarded as complex and voluminous documents that make it harder to extract required information, preventing its smooth functioning. Due to the recent technological advancements, automated and smart contracts can be considered as an alternative to the traditional contract documents that manage its provisions, obligations and clauses by itself. Since the adoption of smart contracts, which can only be performed with the whole participation of involved stakeholders, is limited in the construction industry, this study aims to investigate the stakeholder-associated drivers to implementing smart contracts. For this purpose, a framework based on fuzzy technique for order of preference by the similarityto-ideal-solution (TOPSIS) is developed consisting of eighteen drivers to be prioritized. A number of eighteen experts, who have adequate experience in both construction industry and contract administration, contributed to this study. The results indicate that (i) simple layout to read, (ii) reduction in risks of clients, (iii) clarity in responsibility and risk allocation, (iv) easy to comprehend from various stakeholders, and (v) conflict, claim and dispute reduction were the top five drivers to implement smart contracts. The findings imply that other than its own advantages, the reasons to seek the adoption of smart contracts are mainly the deficiencies of the traditional contract documents. The findings of this study are expected to assist research and development departments of the firms seeking to implement smart contract, while addressing the gap in the literature in this context.
\end{abstract}

\section{Keywords}

Smart contracts; Contract administration; Contract drafting; Fuzzy TOPSIS; Stakeholder perspective

Received: 12 May 2020; Accepted: 19 June 2020

ISSN: 2630-5771 (online) (C) 2020 Golden Light Publishing All rights reserved.

\section{Introduction}

Effective contract administration has significant impact on the achievement of project goals successfully. Project scope, risk allocation, definition of responsibilities and technical details of the required tasks are formed in contracts $[1,2]$, which give legal responsibility to involved parties [3]. Thus, fair and well-designed contracts can be considered as a catalyst to meet expectations of contracting parties [4].
Despite its conservative characteristics, the pace of change in construction industry appears indispensably due to rapid technological advancements. Various forms of automation in construction has been widely examined and adopted in construction projects, but still the key legal document, the contract itself, has not been investigated adequately in terms of automation, yet there is a huge potential [5]. Construction contracts are complex and voluminous documents that make it harder to read and achieve common interpretation principles by numerous involving parties [6]. In

\footnotetext{
Corresponding author

Email: kerimkoc@yildiz.edu.tr
} 
addition, contracts are often drafted by lawyers with legalese language, but read and used by engineers without proper legal knowledge [7]. When the contracts become automated and intelligent, then the involvement of lawyers is no longer at the periphery [5]. Although full automation is extremely challenging, the advantages of automated and smart contract support the efforts made in this regard [8].

There are a huge number of studies investigating contracts with respect to various issues such as claims [9], dispute resolution clauses [10], semantics [11], readability [12] and standard forms [7] etc., however, the adoption of smart contracts is overlooked in the literature. Given this gap, this study is an attempt to investigate stakeholder-associated drivers to implementing smart contracts, highlighting that the effective adoption relies on the willingness of the contracting parties. Fuzzy technique for order of preference by the similarity-to-ideal-solution (TOPSIS) framework was developed and drivers were analysed based on data provided by industry professionals who are experienced in contract administration. Briefly, this study aims to ascertain the most significant reasons that companies would like to adopt smart contracts, in order to assist research and development entities of construction companies in understanding the essential needs about the automation of construction contracts.

\section{Smart contracts in construction industry}

Smart contract can be defined as a computer-based transaction protocol executing contract expressions to satisfy contractual obligations such as payment, liens and enforcement [13]. Contractual performance, payments and other procedures can be embedded as code by rule-based operators in smart contracts, which is irrevocable, once initiated [14].

A construction contract generally consists of three elements as involved parties, intended works, and clauses including provisions. Obligations, permissions, responsibilities, risk allocation and prohibitions are all indicated in contract clauses, through which a contract execution can become automated. One of the most prevailing study and framework about smart contracts was performed by Luo et al. [15]. They developed a semi-automated blockchain-based interim payment framework to ease the manual payment process. A payment logic was formalized to automate actions required prior to the initiation of interim payments and an example scenario was proposed to visualize proposed approach. Mason and Escott [8] examined the perceptions of stakeholders to adopt smart contracts in construction projects. They performed a questionnaire and listed major barriers in smart contract adoption. Expensive drafting process, standardization issues, bugs and cyber threats were considered as some of the reasons of slow implementation. Nanayakkara et al. [16] investigated stakeholders perceptions on blockchain and smart contracts in construction supply chains and found that efficiency, fairness, trust, security, transparency, accountability, compliance and standardization were the major concepts addressed by diverse stakeholder to adopt smart contracts.

\section{Research methodology}

The research methodology followed in this study is provided in Fig. 1. First, stakeholder-associated drivers to adopting smart contracts were identified through literature review. Past studies $[5,8,13,17-$ 19] were used to form the initial list of drivers of smart contracts. A list of drivers was then finessed with a pilot study to ensure that the problem was handled adequately, indicating stakeholder associations. The final list of drivers is provided in Fig. 2. It should be noted that most of the factors that were found through literature survey were revized with respect to stakeholder perspectives. A questionnaire based on fuzzy TOPSIS method was developed and experts were selected by using purposive sampling technique [20] to attend the questionnaire. Data was collected via one-to-one interviews by using linguistic variables, which were then converted to their triangular fuzzy equivalences [21]. 
Determination of stakeholder-associated drivers to implementing smart contracts

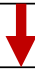

Refinement of the drivers for final arrangement of fuzzy TOPSIS framework with a pilot study

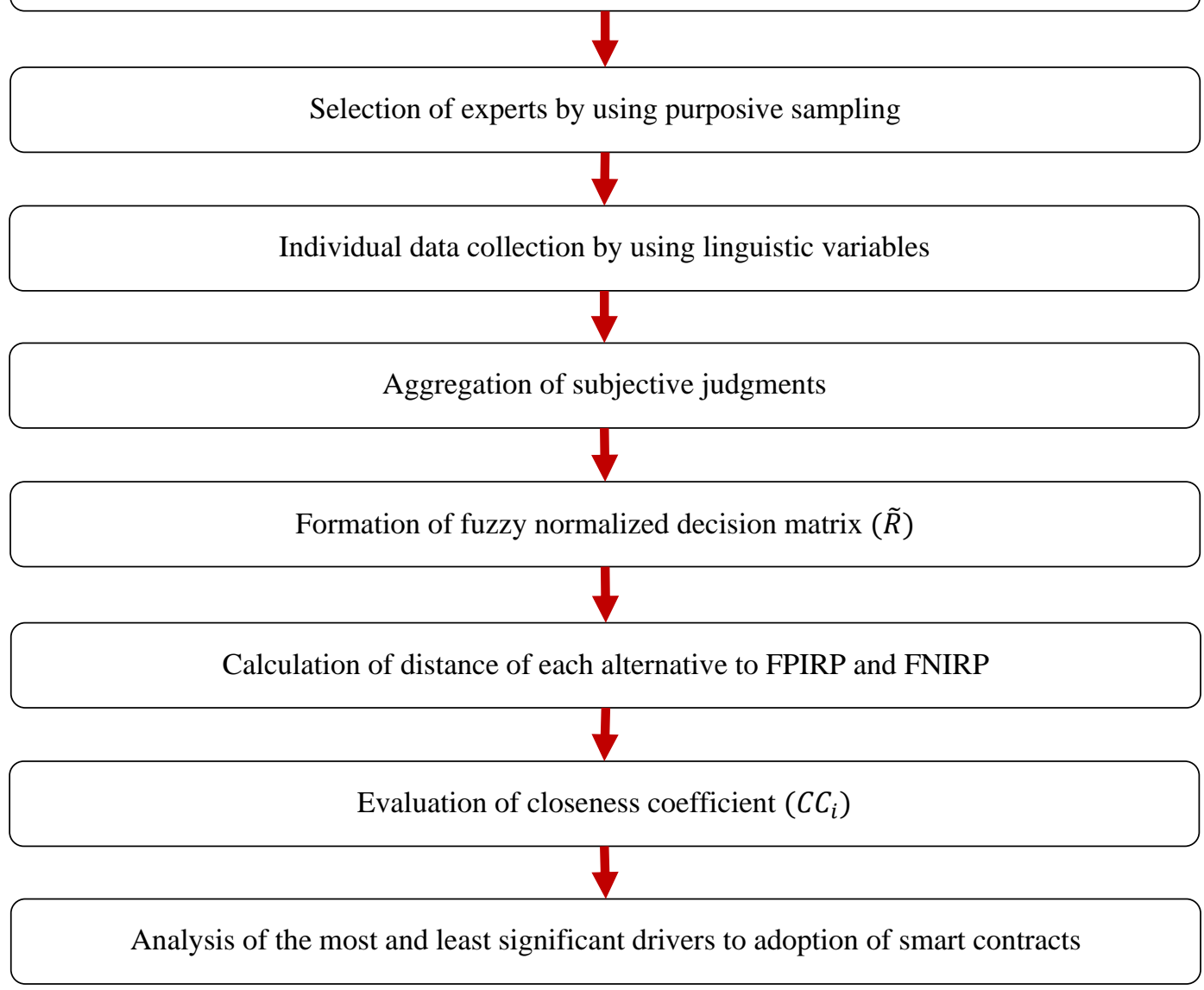

Fig. 1. Research flow

The judgments of experts were aggregated to determine i) fuzzy normalized decision matrix, ii) fuzzy positive ideal reference point (FPIRP) and fuzzy negative ideal reference point (FNIRP), and iii) closeness coefficient. At the final step, drivers were ranked based on closeness coefficients to orient the most significant ones.

\subsection{Expert profile}

Due to the subjective nature of multi-attributedecision-making (MADM) methods, huge number of participants is not required. It was asserted that a large sample size used in such methods could even be impractical, since "cold-called" experts may provide arbitrary answers [22]. Therefore, experts participated to the study were selected carefully based on their experience [23] in both construction industry and contract administration. In this respect, quality of experts rather than quantity was taken into account. Besides, in order to obtain diversity of opinions, all experts were chosen from different companies [24]. The profile of the experts attended to pilot study and questionnaire is given in Table 1 . 3 experts were asked to attend pilot study section of this study, by considering their experiences, involvement in contract related issues, and willingness to aid in the research, all accepted. 
D1: Easy to comprehend from various stakeholders

D2: Simple layout to read

*D3: Reduction in risks of clients

*D4: Reduction in risks of contractors

*D5: Reduction in risks of subcontractors

*D6: Reduction in risks of suppliers

D7: Clarity in responsibility and risk allocation

D8: Conflict, claim and dispute reduction

D9: Adaptability to construction contracts

*D10: Smoother processes in client/contractor relationship

*D11: Smoother processes in contractor/subcontractor relationship

*D12: Smoother functioning in the relationships with suppliers

*D13: Better stakeholder communication

*D14: Expediting procurement processes

*D15: Improvement in the quality of procurement process

*D16: Cost reduction in procurement process

*D17: Client's orientation to adopt smart contracts

*D18: Contractor's orientation to adopt smart contracts

*Drivers were included (or largely changed) during the pilot study.
The most significant driver

\section{Fuzzy} TOPSIS method
The least significant driver

Fig. 2. Framework of the study 
Table 1 Profile of experts

\begin{tabular}{lcccc}
\hline ID & Proficiency & Role & $\begin{array}{c}\text { Experience in } \\
\text { construction industry }\end{array}$ & $\begin{array}{c}\text { Experience in contract } \\
\text { administration }\end{array}$ \\
\hline E1 & Civil engineer & Contract manager & 11 & 8 \\
E2 & Civil engineer & Cost control manager & 20 & 12 \\
E3 & Architect & Technical office coordinator & 17 & 13 \\
*E4 & Civil engineer & Tendering manager & 23 & 23 \\
E5 & Civil engineer & Real estate director & 29 & 29 \\
E6 & Civil engineer & Senior executive director & 20 & 20 \\
$*$ E7 & Architect & Project manager & 38 & 34 \\
E8 & Civil engineer & Interim payment engineer & 11 & 9 \\
E9 & Architect & Design manager & 27 & 15 \\
*E10 & Civil engineer & Project manager & 19 & 19 \\
E11 & Architect & Technical office coordinator & 16 & 16 \\
E12 & Civil engineer & Technical office engineer & 22 & 13 \\
E13 & Civil engineer & Technical office manager & 15 & 7 \\
E14 & Civil engineer & Interim payment engineer & 12 & 10 \\
E15 & Civil engineer & Senior executive director & 25 & 4 \\
E16 & Industrial engineer & Planning engineer & 6 & 15 \\
E17 & Architect & Project architect & 25 & \\
E18 & Industrial engineer & Owner & 25 & 15 \\
\hline$*$ Ex & & & & 25 \\
\hline
\end{tabular}

*Expert attended to pilot study.

Then 28 industry representatives were asked to attend the fuzzy TOPSIS questionnaire (including those attended to pilot study) and 18 of them accepted to attend the fuzzy TOPSIS questionnaire with varying roles in construction companies. Main contractors, clients, consultants and subcontractors were determined to be target groups, however, only limited number of experts from subcontractor and consultant firms were found and invited to the questionnaire survey. Contractual role of the company of the experts is illustrated in Fig. 3. 50\% of the experts were from contractor firms, while nearly $39 \%$ from clients. One expert from subcontractor and consultant firms also contributed to the study. Past literature about the adoption of fuzzy TOPSIS method supports the sample size used in this study [25-28].

\subsection{Fuzzy TOPSIS}

TOPSIS method was first introduced by Hwang and Yoon [29], and is one of the most widely used MADM tools [30]. This study adopted TOPSIS approach due to its several advantageous. First, the sound logic in TOPSIS method is close to the nature of human decision making process [31], since it establishes a solution by considering the shortest distance to the ideal solution and the longest distance to the negative-ideal solution [32]. Stability, mathematical calculation and required computational time can also be regarded as strong attributes of TOPSIS method [33]. Ertuğrul and Karakaşoğlu [34] asserted that TOPSIS is one of the most powerful MADM methods in prioritization problems. 


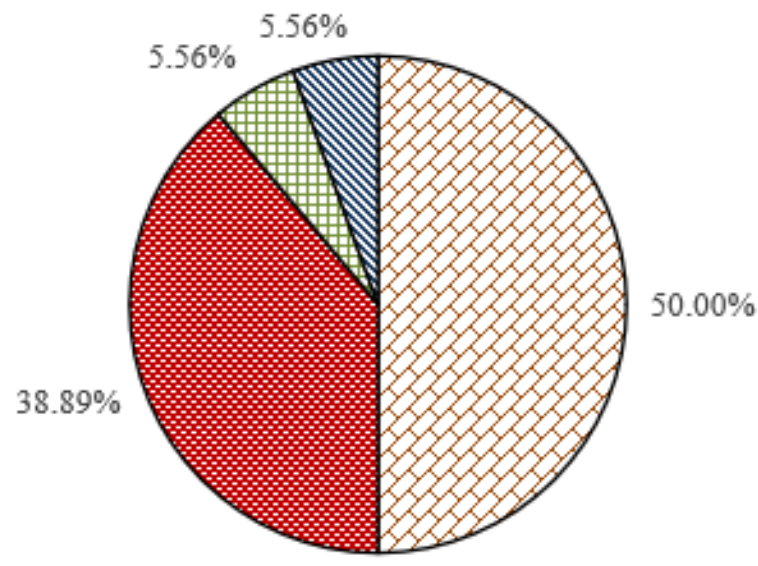

口Contractor $\mathbf{0}$ Client $\mathbf{\theta}$ Subcontractor $\mathbf{\$}$ Consultant

Fig. 3. Contractual role of the experts' company

However, due to the inability of the traditional MADM approaches to deal with fuzziness and uncertainty based on data collected from experts with subjective opinions, fuzzy set theory, introduced by Zadeh [35], needs to be implemented in any selected MADM tool. Therefore, fuzzy TOPSIS approach was considered in this study to deal with possible reliability issues [36]. In fuzzy TOPSIS method, linguistic variables assigned by experts are converted to their triangular equivalences, as shown in Table 2 [37].

Five main steps were used in this study to specify ranking of drivers with respect to fuzzy TOPSIS method. These steps are provided in the followings [38]:

Step 1. Judgments of experts participated to the questionnaire individually were aggregated using Eq. 1.

$\tilde{x}_{i j}=\frac{1}{k}\left[\sum_{e=1}^{k} \tilde{x}_{i j}^{e}\right] \quad i=1,2, \ldots, m ; j=1,2, \ldots, n$

where $\tilde{x}_{i j}=\left(a_{i j}^{k}, b_{i j}^{k}, c_{i j}^{k}\right)$ is a triangular fuzzy number, $k$ is the number of experts, $m$ is the number of alternatives and $n$ is the number of criteria. In other words, arithmetic mean of the judgments was used to indicate the preference of all experts.

Step 2. Fuzzy normalized decision matrix $(\tilde{R})$ was formed using Eqs. 2-4.

$$
\tilde{R}=\left[\tilde{r}_{i j}\right]_{m x n} \quad i=1,2, \ldots, m ; j=1,2, \ldots, n
$$

$\tilde{r}_{i j}=\left(\frac{a_{i j}}{c_{j}^{+}}, \frac{b_{i j}}{c_{j}^{+}}, \frac{c_{i j}}{c_{j}^{+}}\right)$

$c_{j}^{+}=\max c_{i j}$

where $\tilde{r}_{i j}$ is a normalized triangular fuzzy number $\left(r a_{i j}, r b_{i j}, r c_{i j}\right)$ for each alternative. It means that each element in triangular fuzzy numbers was divided by maximum possible value in the corresponding matrix to calculate normalized values.

Step 3. Fuzzy weighted normalized decision matrix $(\tilde{V})$ is formed through Eqs. 5-6.

$\tilde{V}=\left[\tilde{v}_{i j}\right]_{m x n} \quad i=1,2, \ldots, m ; j=1,2, \ldots, n$

$\tilde{v}_{i j}=\tilde{r}_{i j} \otimes \widetilde{w}_{j}$

where $\widetilde{w}_{j}$ represents the relative weight of criterion $C_{j}$,

Step 4. The distance to the fuzzy positive ideal reference point (FPIRP, $A^{+}$) and fuzzy negative ideal reference point (FNIRP, $A^{-}$) are determined via Eqs. 7-8. 
Table 2. Linguistic variables for criteria weights and alternatives

\begin{tabular}{llll}
\hline Linguistic variables & $\begin{array}{l}\text { Corresponding triangular } \\
\text { fuzzy number }\end{array}$ & Linguistic variables & $\begin{array}{l}\text { Corresponding triangular } \\
\text { fuzzy number }\end{array}$ \\
\hline Importance weights & $(0,0,0.1)$ & Rankings \\
Very low (1) & $(0,0.1,0.3)$ & Very poor $(2)$ & $(0,0,1)$ \\
Low (2) & $(0.1,0.3,0.5)$ & Poor $(3)$ & $(0,1,3)$ \\
Medium low (3) & $(0.3,0.5,0.7)$ & Fair $(4)$ & $(1,3,5)$ \\
Medium (4) & $(0.5,0.7,0.9)$ & Good $(5)$ & $(3,5,7)$ \\
Medium high (5) & $(0.7,0.9,1.0)$ & Very good $(6)$ & $(5,7,9)$ \\
High (6) & $(0.9,1.0,1.0)$ & Excellent $(7)$ & $(7,9,10)$ \\
Very high (7) & & & $(9,10,10)$ \\
\hline
\end{tabular}

$d_{i}^{+}=\sum_{j=1}^{n} d\left(\tilde{v}_{i j}, \tilde{v}_{j}^{+}\right)$

$d_{i}^{-}=\sum_{j=1}^{n} d\left(\tilde{v}_{i j}, \tilde{v}_{j}^{-}\right)$

With respect to Eqs. 9-10.

$A^{+}=\left(\tilde{v}_{1}^{+}, \tilde{v}_{2}^{+}, \ldots, \tilde{v}_{n}^{+}\right)$

$A^{-}=\left(\tilde{v}_{1}^{-}, \tilde{v}_{2}^{-}, \ldots, \tilde{v}_{n}^{-}\right)$

where $d_{i}^{+}$denotes the distance of alternative $i$ from FPIRP, and $d_{i}^{-}$denotes the distance of alternative $i$ from FNIRP. $\tilde{v}_{j}^{+}=(1,1,1)$ and $\tilde{v}_{j}^{-}=(0,0,0)$, since the triangular fuzzy numbers are in the range between 0 and 1 .

$d(\tilde{A}, \tilde{B})$ denotes the distance between two fuzzy numbers and can be calculated as:

$d(\tilde{A}, \tilde{B})=\sqrt{\frac{\left[\left(a_{1}-b_{1}\right)^{2}+\left(a_{2}-b_{2}\right)^{2}+\left(a_{3}-b_{3}\right)^{2}\right]}{3}}$

Step 5. Closeness coefficient $\left(C C_{i}\right)$ are calculated at the last step of fuzzy TOPSIS method.

$C C_{i}=\frac{d_{i}^{-}}{d_{i}^{+}+d_{i}^{-}}$

The higher the value $C C_{i}$ is, closer to the FPIRP and farther to FNIRP of the alternative $i$. It means that high value of $C C_{i}$ implies a good performance of alternative $i$. Note that the alternatives in this study are stakeholder-associated drivers.

\section{Results and discussion}

Fuzzy TOPSIS methodology was adopted in this study to rank stakeholder-associated drivers to implement smart contracts. Fuzzy decision matrix calculated based on the judgments of 18 experts, is shown in Table 3. Then normalized decision matrix was formed (Table 4). The results of fuzzy TOPSIS analysis are given in Table 5 .

Table 3 Fuzzy decision matrix

\begin{tabular}{lccc}
\hline Drivers & $a_{i j}$ & $b_{i j}$ & $c_{i j}$ \\
\hline D1 & 6.22 & 7.89 & 9.00 \\
D2 & 6.67 & 8.39 & 9.50 \\
D3 & 6.56 & 8.28 & 9.33 \\
D4 & 5.61 & 7.39 & 8.61 \\
D5 & 4.89 & 6.61 & 8.11 \\
D6 & 5.17 & 6.89 & 8.39 \\
D7 & 6.56 & 8.22 & 9.28 \\
D8 & 5.94 & 7.50 & 8.61 \\
D9 & 4.44 & 6.17 & 7.61 \\
D10 & 5.56 & 7.39 & 8.78 \\
D11 & 4.11 & 5.67 & 7.00 \\
D12 & 4.22 & 5.83 & 7.28 \\
D13 & 5.56 & 7.33 & 8.72 \\
D14 & 5.00 & 6.83 & 8.39 \\
D15 & 5.50 & 7.11 & 8.39 \\
D16 & 3.83 & 5.44 & 7.06 \\
D17 & 4.00 & 5.56 & 7.00 \\
D18 & 3.22 & 4.72 & 6.22 \\
\hline
\end{tabular}


Table 4 Fuzzy normalized decision matrix

\begin{tabular}{lccc}
\hline Drivers & $r a_{i j}$ & $r b_{i j}$ & $r c_{i j}$ \\
\hline D1 & 0.655 & 0.830 & 0.947 \\
D2 & 0.702 & 0.883 & 1.000 \\
D3 & 0.690 & 0.871 & 0.982 \\
D4 & 0.591 & 0.778 & 0.906 \\
D5 & 0.515 & 0.696 & 0.854 \\
D6 & 0.725 & 0.883 \\
D7 & 0.544 & 0.865 & 0.977 \\
D8 & 0.690 & 0.789 & 0.906 \\
D9 & 0.626 & 0.649 & 0.801 \\
D10 & 0.778 & 0.924 \\
D11 & 0.596 & 0.737 \\
D12 & 0.468 & 0.614 & 0.766 \\
D13 & 0.585 & 0.772 & 0.918 \\
D14 & 0.433 & 0.719 & 0.883 \\
D15 & 0.444 & 0.749 & 0.883 \\
D16 & 0.585 & 0.573 & 0.743 \\
D17 & 0.526 & 0.585 & 0.737 \\
D18 & 0.579 & 0.497 & 0.655 \\
\hline
\end{tabular}

Table 5 The results of fuzzy TOPSIS analysis

\begin{tabular}{llcccl}
\hline ID & Drivers & $d_{i}^{+}$ & $d_{i}^{-}$ & \multicolumn{1}{c}{$C C_{i}$} & Rank \\
\hline D1 & Easy to comprehend from various stakeholders & 2.224 & 0.820 & 0.269 & 4 \\
D2 & Simple layout to read & 2.185 & 0.870 & 0.285 & 1 \\
D3 & Reduction in risks of clients & 2.194 & 0.856 & 0.281 & 2 \\
D4 & Reduction in risks of contractors & 2.274 & 0.769 & 0.253 & 8 \\
D5 & Reduction in risks of subcontractors & 2.341 & 0.702 & 0.231 & 12 \\
D6 & Reduction in risks of suppliers & 2.315 & 0.731 & 0.240 & 10 \\
D7 & Clarity in responsibility and risk allocation & 2.196 & 0.852 & 0.280 & 3 \\
D8 & Conflict, claim and dispute reduction & 2.254 & 0.782 & 0.258 & 5 \\
D9 & Adaptability to construction contracts & 2.386 & 0.654 & 0.215 & 13 \\
D10 & Smoother processes in client/contractor relationship & 2.275 & 0.775 & 0.254 & 6 \\
D11 & Smoother processes in contractor/subcontractor relationship & 2.430 & 0.602 & 0.198 & 15 \\
D12 & Smoother functioning in the relationships with suppliers & 2.413 & 0.622 & 0.205 & 14 \\
D13 & Better stakeholder communication & 2.278 & 0.770 & 0.253 & 7 \\
D14 & Expediting procurement processes & 2.325 & 0.724 & 0.238 & 11 \\
D15 & Improvement in the quality of procurement process & 2.291 & 0.747 & 0.246 & 9 \\
D16 & Cost reduction in procurement process & 2.449 & 0.590 & 0.194 & 17 \\
D17 & Client's orientation to adopt smart contracts & 2.438 & 0.595 & 0.196 & 16 \\
D18 & Contractor's orientation to adopt smart contracts & 2.519 & 0.514 & 0.169 & 18 \\
\hline
\end{tabular}


The results indicate that "simple layout to read (D2, 0.285)", "reduction in risks of clients (D3, $0.281)$ ", "clarity in responsibility and risk allocation (D7, 0.280)", "easy to comprehend from various stakeholders (D1, 0.269)" and "conflict, claim and dispute reduction (D8, 0.258)" were the top five drivers to adopting smart contracts.

It is unexpected that D2 was found to be the most significant driver, since readability issues in encoded expressions can also be regarded as one of the barriers to implementing smart contracts. Even standardization and familiarity are shown as preliminary steps for the smooth functioning of smart contracts [8]. The main reason of this could be the current readability issues of traditional type of contracts, since construction contracts are always blamed to be long, complex and hard to read documents [6]. D3 was ranked as the second most significant driver. It is an estimated finding since most of time the drafting process is held by clients, and they can minimize their risks and orient the contracts by their own interests by adopting smart contracts, occasionally with the help of consultants. However, this could impose a high risk for other contracting parties such as contractors and subcontractors. D7 was the third most significant driver, which highlight the importance of clear risk allocation and responsibility. This can also be associated with the ambiguous traditional contract documents, since unclear responsibilities and improper risk allocation could entail conflicts [39]. If drafted properly, smart contracts can guarantee the clear obligations and responsibilities. D1 was considered as the fourth most significant driver indicating the expectations of divergent stakeholders from smart contracts about comprehensiveness. Finally, D8 was the fifth most significant driver, which emphasizes the inherent nature of construction industry with a massive number of conflicts, claims, and unresolved disputes. In overall view, the findings indicate that industry professionals expect from smart contracts to reduce contractual conflicts, comprehensiveness, clear responsibilities, and simple layout.

Meanwhile, "contractor's orientation to adopt smart contracts (D18, 0.169)" was the least significant driver to adopting smart contracts. Contractors may consider that they can be faced with various risks by client-based drafting process of smart contracts, thus may show clear resistance towards smart contracts. "Cost reduction in procurement process (D16, 0.194)" and "client's orientation to adopt smart contracts (D17, 0.196)" were the second and third least significant drivers that force industry to implement smart contracts. It means that there would not be enough cost reduction in procurement process that attract the industry for smart contract adoption, in spite of the fact that digitization could reduce transaction cost [17]. The reason of this could be that direct cost would remain constant, but the indirect cost could be reduced, and process might be relieved via technological improvements in contracts. One of the most significant findings of this study was that even though clients' risks can be reduced, still there is not enough client willingness to adopt smart contracts.

\section{Conclusions}

Contracts that give a legal responsibility to initiate intended works have significant impact on the successful project execution phase. Numerous risks within the contract document threaten the effective contract administration leading to adversarial relationships among contracting parties. Due to the rapid technological advancements, automated contracts can be considered as an alternative to the traditional contract documents that manage its provisions, obligations and clauses itself. In line with these, this study aims to evaluate stakeholderassociated drivers to implementing smart contracts by using fuzzy technique for order of preference by the similarity-to-ideal-solution method.

Decision framework was developed through literature survey and corrected with a pilot study, since there are only few studies investigated the adaptation of smart contracts to construction industry. The findings imply that simple layout to read, reduction in risks of clients, clarity in responsibility and risk allocation, easy to comprehend from various stakeholders, and conflict, claim and dispute reduction were the top 
five drivers to implement smart contracts. The results highlight that the reason to adopt smart contract could be various deficiencies of traditional contract documents, instead of the benefits of smart contracts. It was found that risks of clients and conflicts can be reduced, responsibilities can be clarified, and contracts can become easier to read and understand with the adoption of smart contracts. Despite reduction in risks of client was ranked at the top, clients' orientation to adopt smart contracts was found to be among the least significant drivers. This controversy indicates the resistance of the clients to adopt smart contracts despite its obvious superiority over traditional contract documents.

The findings obtained may increase the awareness on the smart contracts in the construction industry. Top drivers determined in this study can be focused by research and development teams of construction firms to serve as much benefits as possible prior to smart contract drafting processes. Possible solutions to the least significant drivers can also reasonably improve the willingness to adopt smart contracts. Particular risks in smart contracts can be investigated in future studies.

\section{Declaration of conflicting interests}

The author(s) declared no potential conflicts of interest with respect to the research, authorship, and/or publication of this article.

\section{References}

[1] Nguyen DA, Garvin MJ, Gonzalez EE (2018). Risk Allocation in U.S. Public-Private Partnership Highway Project Contracts. Journal of Construction Engineering and Management 144(5):04018017.

[2] Zhang SB, Fu YF, Gao Y, Zheng XD (2016). Influence of trust and contract on dispute negotiation behavioral strategy in construction subcontracting. Journal of Management in Engineering 32(4):04016001.

[3] Mitropoulos P, Howell G (2001). Model for Understanding, Preventing, and Resolving Project Disputes. Journal of Construction Engineering and Management 127(3):223-231.
[4] Lee J, Ham Y, Yi JS, Son J (2020). Effective Risk Positioning through Automated Identification of Missing Contract Conditions from the Contractor's Perspective Based on FIDIC Contract Cases. Journal of Management in Engineering 36(3):05020003.

[5] Mason J (2017). Intelligent Contracts and the Construction Industry. Journal of Legal Affairs and Dispute Resolution in Engineering and Construction 9(3):04517012.

[6] Hassan FU, Le T (2020). Automated Requirements Identification from Construction Contract Documents Using Natural Language Processing. Journal of Legal Affairs and Dispute Resolution in Engineering and Construction 12(2):04520009.

[7] Besaiso H, Fenn P, Emsley M, Wright D (2018). A comparison of the suitability of FIDIC and NEC conditions of contract in Palestine: A perspective from the industry. Engineering, Construction and Architectural Management 25(2):241-256.

[8] Mason J, Escott H (2018). Smart Contracts in Construction: Views and Perceptions of Stakeholders. Proc. FIG Conf. 8-11 May 2018, Istanbul, Turkey .

[9] Zaneldin EK (2018). Investigating the types, causes and severity of claims in construction projects in the UAE. International Journal of Construction Management 1:1-17.

[10] Jagannathan M, Delhi VSK (2019). Litigation Proneness of Dispute Resolution Clauses in Construction Contracts. Journal of Legal Affairs and Dispute Resolution in Engineering and Construction 11(3):04519011.

[11] Youssef A, Osman H, Georgy M, Yehia N (2018). Semantic Risk Assessment for $\mathrm{Ad} \mathrm{Hoc} \mathrm{and}$ Amended Standard Forms of Construction Contracts. Journal of Legal Affairs and Dispute Resolution in Engineering and Construction 10(2):04518002.

[12] Rameezdeen R, Rajapakse C (2007). Contract interpretation: The impact of readability. Construction Management and Economics 25(7):729-737.

[13] Szabo N (1994). Smart Contracts. Available from: http://www.fon.hum.uva.nl/rob/Courses/Informati onInSpeech/CDROM/Literature/LOTwinterschool 2006/szabo.best.vwh.net/smart.contracts.html, [05/05/2020].

[14] Norton Rose Fulbright (2016). Smart Contracts: coding the fine print. Available from: https://www.nortonrosefulbright.com/en/knowledg 
e/publications/9c35a32f/smart-contracts-codingthe-fine-print, [05/05/2020].

[15] Luo H, Das M, Wang J, Cheng JCP, Kong H (2019). Construction Payment Automation through Smart Contract-based Blockchain Framework. 36th Int. Symp. Autom. Robot. Constr. 21-24 May 2019, Alberta, Canada .

[16] Nanayakkara S, Perera S, Senaratne S (2019). Stakeholders' Perspective on Blockchain and Smart Contracts Solutions for Construction Supply Chains. CIB World Build. Congr. 17-21 June 2019, Hong Kong, China .

[17] Mason J (2019). BIM Fork: Are Smart Contracts in Construction More Likely to Prosper with or without BIM? Journal of Legal Affairs and Dispute Resolution in Engineering and Construction 11(4):02519002.

[18] Nanayakkara S, Perera S, Senaratne S (2019). Stakeholders ' Perspective on Blockchain and Smart Contracts Solutions for Construction Supply Chains. CIB World Build. Congr. .

[19] Perera S, Nanayakkara S, Rodrigo MNN, Senaratne S, Weinand R (2020) Blockchain technology: Is it hype or real in the construction industry? Journal of Industrial Information Integration 17(June 2019):100125.

[20] Budayan C (2019). Evaluation of Delay Causes for BOT Projects Based on Perceptions of Different Stakeholders in Turkey. Journal of Management in Engineering 35(1):04018057.

[21] Jokar E, Aminnejad B, Lorak A (2020). Risk prioritization and selection of contractor participating in Public- Private Partnership ( PPP ) infrastructure project using Hybrid Fuzzy AHP and Fuzzy TOPSIS ( Case Study : Saveh-Salafchegan Freeway Project ). Journal of Construction Engineering, Management \& Innovation 3(1):116.

[22] Darko A, Ping A, Chan C, Ameyaw EE, Owusu K, Pärn E, Edwards DJ, Darko A, Ping A, Chan C, Ameyaw EE (2019). Review of application of analytic hierarchy process (AHP) in construction. International Journal of Construction Management 19(5):436-452.

[23] Arslan V, Ulubeyli S (2019). Sorting at source and reusing: The case of construction and demolition waste in Turkey. Journal of Construction Engineering, Management \& Innovation 2(4):230236.

[24] Ozorhon B, Kus C, Caglayan S (2020). Assessing competitiveness of international contracting firms from the managerial perspective by using Analytic Network Process. Journal of Construction Engineering, Management \& Innovation 3(1):5266.

[25] Jang W, Hong HU, Han SH, Baek SW (2017). Optimal Supply Vendor Selection Model for LNG Plant Projects Using Fuzzy-TOPSIS Theory. Journal of Management in Engineering 33(2):04016035.

[26] Liang R, Zhang J, Wu C, Sheng Z, Wang X (2019). Joint-Venture Contractor Selection Using Competitive and Collaborative Criteria with Uncertainty. Journal of Construction Engineering and Management 145(2):04018123.

[27] Rostamzadeh R, Ghorabaee MK, Govindan K, Esmaeili A, Nobar HBK (2018). Evaluation of sustainable supply chain risk management using an integrated fuzzy TOPSIS- CRITIC approach. Journal of Cleaner Production 175:651-669.

[28] Gupta H, Barua MK (2017). Supplier selection among SMEs on the basis of their green innovation ability using BWM and fuzzy TOPSIS. Journal of Cleaner Production 152:242-258.

[29] Hwang CI, Yoon K (1981). Multiple Attribute Decision Making. Berlin: Springer-Verlag.

[30] Polat G, Turkoglu H, Damci A, Demirli I (2019). A comparative study on selecting urban renewal project via different MADM methods. Journal of Construction Engineering, Management \& Innovation 2(3):131-143.

[31] Kim G, Park CS, Yoon KP (1997). Identifying investment opportunities for advanced manufacturing systems with comparativeintegrated performance measurement. International Journal of Production Economics 50(1):23-33.

[32] Opricovic S, Tzeng GH (2004). Compromise solution by MCDM methods: A comparative analysis of VIKOR and TOPSIS. European Journal of Operational Research 156(2):445-455.

[33] Iç YT (2012). An experimental design approach using TOPSIS method for the selection of computer-integrated manufacturing technologies. Robotics and Computer-Integrated Manufacturing 28(2):245-256.

[34] Ertuğrul I, Karakaşoğlu N (2008). Comparison of fuzzy AHP and fuzzy TOPSIS methods for facility location selection. International Journal of Advanced Manufacturing Technology 39(78):783-795.

[35] Zadeh LA (1965). Fuzzy Sets. Information and Control 8:338-353. 
[36] Gurgun AP, Koc K (2020). Contractor prequalification for green buildings-evidence from Turkey. Engineering, Construction and Architectural Management 27(6):1377-1400.

[37] Chen CT (2000) Extensions of the TOPSIS for group decision-making under fuzzy environment. Fuzzy Sets and Systems 114(1):1-9.

[38] Wang TC, Chang TH (2007). Application of TOPSIS in evaluating initial training aircraft under a fuzzy environment. Expert Systems with Applications 33(4):870-880.

[39] Schuhmann R, Eichhorn B (2017). Reconsidering contact risk and contractual risk management. International Journal of Law and Management 59(4):504-521. 